\title{
OPEN Transketolase regulates sensitivity to APR-246 in p53-null cells independently of oxidative stress modulation
}

\author{
Julia V. Milne ${ }^{1,2}$, Bonnie Z. Zhang ${ }^{1,2}$, Kenji M. Fujihara ${ }^{1,2}$, Swati Dawar ${ }^{1,2}$, \\ Wayne A. Phillips ${ }^{1,2,3}$ \& Nicholas J. Clemons ${ }^{1,2} \llbracket$
}

The prevalence and dire implications of mutations in the tumour suppressor, p53, highlight its appeal as a chemotherapeutic target. We recently showed that impairing cellular antioxidant systems via inhibition of SLC7A11, a component of the system $x_{c}{ }^{-}$cystine-glutamate antiporter, enhances sensitivity to mutant-p53 targeted therapy, APR-246. We investigated whether this synergy extends to other genes, such as those encoding enzymes of the pentose phosphate pathway (PPP).TKT, one of the major enzymes of the PPP, is allegedly regulated by NRF2, which is in turn impaired by accumulated mutant-p53 protein. Therefore, we investigated the relationship between mutant-p53, TKT and sensitivity to APR-246. We found that mutant-p53 does not alter expression of TKT, nor is TKT modulated directly by NRF2, suggesting a more complex mechanism at play. Furthermore, we found that in p53null cells, knockdown of TKT increased sensitivity to APR-246, whilst TKT overexpression conferred resistance to the drug. However, neither permutation elicited any effect on cells overexpressing mutant-p53 protein, despite mediating oxidative stress levels in a similar fashion to that in p53-null cells. In sum, this study has unveiled TKT expression as a determinant for sensitivity to APR-246 in p53-null cells.

The tumour suppressor, p53, the 'guardian of the genome', lies at the crux of a complex transcriptional network of proteins involved in maintaining genome integrity ${ }^{1,2}$. Mutation of p53 frequently results in loss of its direct transcriptional ability and deregulation of its tumour suppression pathways ${ }^{1,3,4}$, and can lead to accumulation of mutant 553 protein with gain-of-function oncogenic activity ${ }^{5-11}$.

Targeting mutant or otherwise deregulated p53 is an attractive avenue for cancer therapeutics due to the frequency of such permutations in cancer. As such, numerous strategies have been developed to reactivate mutp53, including inducing conformational changes in mut-p53 to restore its wild-type function. This effect has been demonstrated with the use of the first-in-class small molecule drug, APR-246 (also known as PRIMA- $\left.{ }^{\text {Met }}\right)^{12}$, which is currently showing promise in clinical trials ${ }^{13}$

APR-246 is hydrolysed into its active form, methylene quinuclidinone (MQ), inside cells where it interacts directly with cysteine residues in the $\mathrm{p} 53$ protein $^{4,12,14}$. A subsequent change to the mutant p53 protein conformation restores its tumour-suppressing functions, namely induction of apoptosis via transactivation of target genes PUMA, NOXA and $B A X^{12,15}$. APR-246 potentially also abrogates any oncogenic gain-of-function activity acquired by mut-p53.

In addition, APR-246 has been found to interfere with cellular antioxidant pathways involving glutathione and thioredoxin reductase ${ }^{16-18}$ by the formation of adducts between MQ and cysteine ${ }^{15}$ or selenocysteine ${ }^{17}$, respectively, thereby reducing the antioxidant capacities of the cell. Thus, APR-246 encourages an unfavourably high oxidative stress environment that ultimately results in induction of cell death pathways ${ }^{15-17}$. Importantly, accumulation of mut-p53 protein interferes with the cellular redox response through impairment of the key

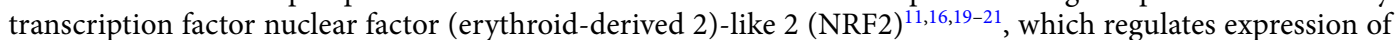
many antioxidant-related genes including thioredoxin reductase and key enzymes in the de novo glutathione synthesis pathway. Thus, mutant p53 itself provides cell selectivity for the oxidative stress-mediated effects of

${ }^{1}$ Peter MacCallum Cancer Centre, 305 Grattan St, Melbourne, VIC 3000, Australia. ${ }^{2}$ Sir Peter MacCallum Department of Oncology, The University of Melbourne, Parkville, VIC 3010, Australia. ${ }^{3}$ Department of Surgery (St Vincent's Hospital), The University of Melbourne, Parkville, VIC 3010, Australia. ${ }^{\boxplus}$ email: nicholas.clemons@ petermac.org 


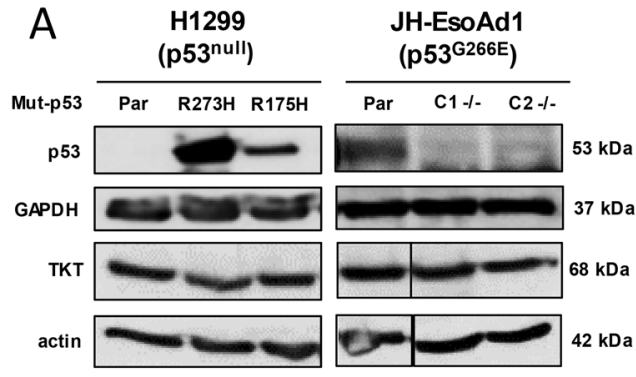

C

H1299

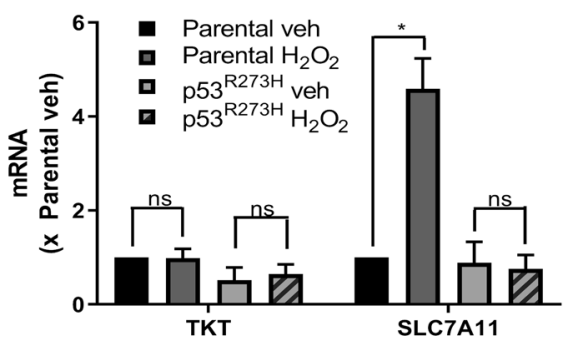

B

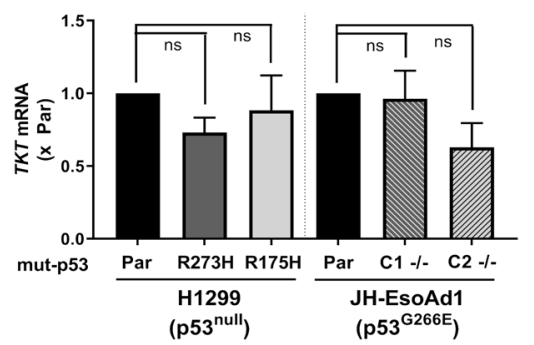

$\mathrm{D}$ H1299

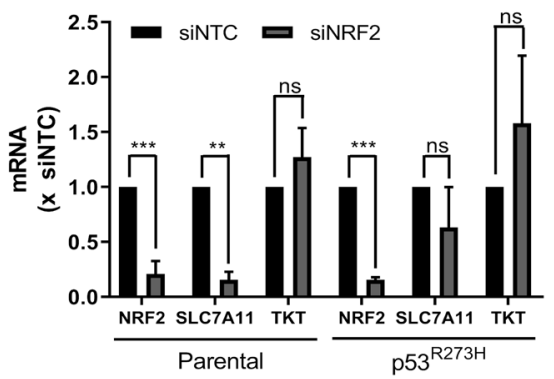

Figure 1. TKT is not regulated by mutant-p53 or NRF2 activation. (A) Expression of p53 and TKT protein in H1299 and JH-EsoAd1 cells (Par, parental; C1 -/-, p53-/- clone 1; C2 -/-, p53-/- clone 2). (B) TKT mRNA (bottom) in H1299 and JH-EsoAd1 cells, with mRNA expression normalised to parental cells (Par, parental; C1 -/-, p53-/- clone 1; C2 -/-, p53-/- clone 2). (C) TKT and SLC7A11 mRNA expression in H1299 parental ( $\left.\mathrm{p} 53^{\text {null }}\right)$ and $\mathrm{p} 53^{\mathrm{R} 273 \mathrm{H}}$ cells following treatment with hydrogen peroxide $\left(\mathrm{H}_{2} \mathrm{O}_{2}, 50 \mu \mathrm{M}\right)$ for $3 \mathrm{~h}$, normalised to parental vehicle treatment. (D) NRF2, SLC7A11 and TKT mRNA expression in H1299 parental (p53 ${ }^{\text {null }}$ ) and $\mathrm{p} 53^{\mathrm{R} 273 \mathrm{H}}$ cells following $48 \mathrm{~h}$ NRF2 knockdown with siRNA, normalised to siNTC (siNTC, non-targeting control; siNRF2, NRF2 siRNA). Data represent mean, error bars $=\mathrm{SEM}, \mathrm{n} \geq 3$ independent experiments for all studies excluding (A) where blots are representative of 2 independent experiments. (B,C) Randomised block one-way ANOVA on raw data with Greenhouse-Geisser correction and Dunnett's multiple comparison posttest. (D) Unpaired Student's $t$-test on raw data. ns, not significant; ${ }^{*} p<0.05$; ${ }^{* *} p<0.01$; ${ }^{* *} p<0.001$.

APR-246 through promoting a pro-oxidative state within the cell and/or impairing feedback responses that combat oxidative stress ${ }^{19,20}$.

NADPH is a crucial factor in the recycling of cellular antioxidants such as the enzymatic reduction of oxidised glutathione (GSSG) to reduced glutathione (GSH) by glutathione reductase, and also in the recycling of thioredoxin via thioredoxin reductase $\mathrm{e}^{17,22,23}$. One of the major sources of NADPH in the cell is the pentose phosphate pathway (PPP) $)^{24,25}$. Transketolase (TKT), encoded by the TKT gene, is a multifunctional enzyme in the non-oxidative arm of the PPP that serves to produce ribose-5-phosphate, a precursor molecule for nucleotide synthesis, as well as channelling its intermediates back into glycolysis in response to oxidative stress and demand for $\mathrm{NADPH}^{25}$.

It has been proposed that TKT expression, like many other antioxidant-related genes, is regulated by NRF2 $2^{25,26}$ and TKT is overexpressed in many tumour types ${ }^{27}$. It follows that TKT has been found to influence the cellular redox balance, where its knockdown causes an accumulation of intracellular ROS, sensitising tumour cells to radio- and chemotherapies ${ }^{25}$. Therefore, we hypothesised that (i) TKT levels would be regulated by accumulation of mut-p53 through its interaction with NRF2, and (ii) that TKT expression would be an important consideration in determining response to APR-246.

\section{Results}

TKT is not regulated by mutant-p53 or NRF2 activation. To investigate the effect of mut-p53 on transketolase (TKT) expression, two isogenic cell systems were used. H1299 cells, a non-small cell lung cancer line, harbour a mutation that prevents the formation of full-length active p53 protein ${ }^{28}$. These cells were previously transduced to ectopically overexpress common missense mutant p53 proteins, p53 ${ }^{\mathrm{R} 273 \mathrm{H}}$ or p53 ${ }^{\mathrm{R} 175 \mathrm{H} 16}$. $\mathrm{JH}-\mathrm{EsoAd} 1$ cells are an oesophageal cancer cell line that endogenously express missense mutant $\mathrm{p} 53^{\mathrm{G} 266 \mathrm{E} 29}$, and two $\mathrm{p} 53^{-/-}$clones were previously generated using CRISPR-Cas $9^{16}$. RT-qPCR and western blotting showed no consistent relationship between TKT mRNA or protein expression, respectively, and the presence or absence of mut-p53 protein (Fig. 1A,B). To test the effect of NRF2 activation on TKT expression, H1299 cells were treated with hydrogen peroxide $\left(\mathrm{H}_{2} \mathrm{O}_{2}\right)$ and mRNA expression analysed by RT-qPCR. Upon $\mathrm{H}_{2} \mathrm{O}_{2}$ treatment and subsequent NRF2 activation (Supplementary Fig. 1A), no change was observed in TKT expression in either H1299 p53 $3^{\text {null }}$ or p53 ${ }^{\mathrm{R} 273 \mathrm{H}}$ cells (Fig. 1C). As a control, expression of SLC7A11, a known NRF2 target gene, was also ana- 
A

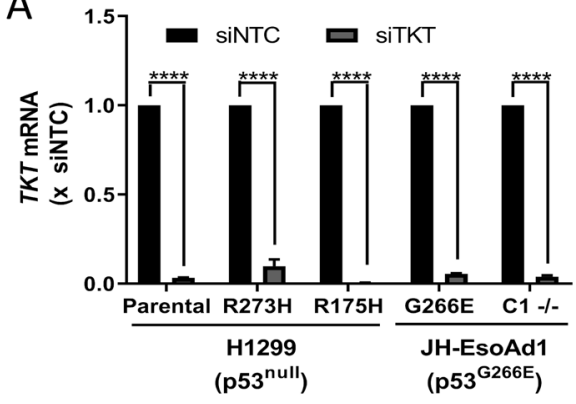

B

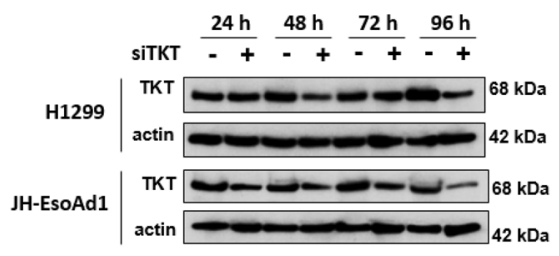

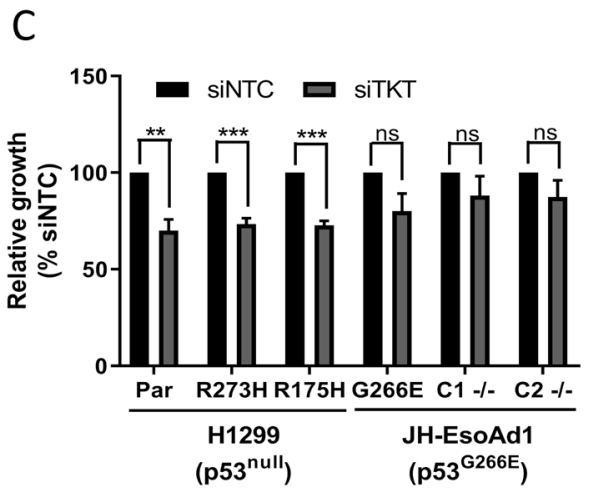

D

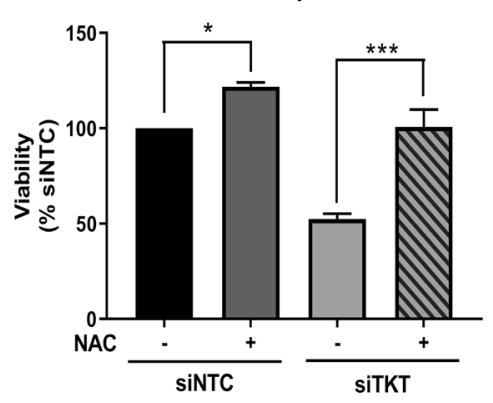

$E$

H1299 $p 53^{\text {null }}$

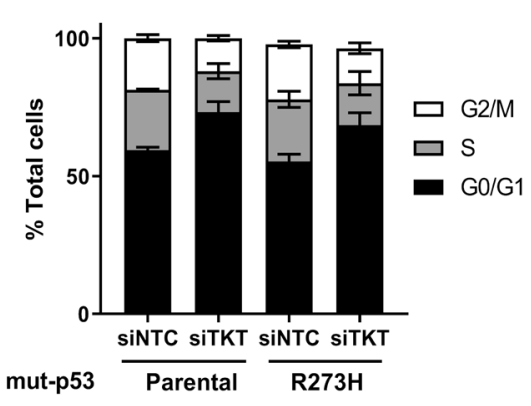

Figure 2. Transient knockdown of TKT slows cell growth and induces cell cycle arrest in H1299 cells irrespective of $\mathrm{p} 53$ status. (A) TKT mRNA expression in $\mathrm{H} 1299$ parental (p53 ${ }^{\text {null }}$ ), $\mathrm{p} 53^{\mathrm{R} 273 \mathrm{H}}$ and $\mathrm{p} 53^{\mathrm{R} 175 \mathrm{H}}$ cells and JH-EsoAd1 parental ( $\mathrm{p} 53^{\mathrm{G} 266 \mathrm{E}}$ ) and p53-/- clone $1(\mathrm{C} 1-/-)$ following $24 \mathrm{~h}$ TKT knockdown with siRNA, normalised to siNTC (siNTC, non-targeting control; siTKT, TKT siRNA). (B) Western blots for TKT in H1299 and JH-EsoAd1 parental cells following TKT knockdown with siRNA for 24, 48, 72 and $96 \mathrm{~h}$, showing actin as a loading control (C) Relative growth of H1299 parental (Par, $\left.\mathrm{p} 53^{\text {null }}\right), \mathrm{p} 53^{\mathrm{R} 273 \mathrm{H}}$ and $\mathrm{p} 53^{\mathrm{R} 175 \mathrm{H}}$ cells and JH-EsoAd1 parental (p53 $\left.{ }^{\mathrm{G} 266 \mathrm{E}}\right), \mathrm{p} 53-/-$ clones $1(\mathrm{C} 1-/-)$ and $2(\mathrm{C} 2-/-)$ following $96 \mathrm{~h}$ TKT knockdown with siRNA. (D) H1299 parental (p53 ${ }^{\text {null }}$ ) cells +/- N-acetyl-cysteine (NAC, $5 \mathrm{mM}$ ) -/-) following $96 \mathrm{~h}$ TKT knockdown with siRNA. (C,D) Data are expressed as a percentage of siNTC. (E) Changes in cell cycle distribution in H1299 parental (p53 null) and p53 $3^{\text {R273H }}$ cells following $96 \mathrm{~h}$ TKT knockdown with siRNA. Data represent mean, error bars $=$ SEM, $n=3$ for all studies excluding $(\mathbf{B}, \mathbf{E})$ where blots and cell cycle analysis are representative of 2 independent experiments. $(\mathbf{A}, \mathbf{C})$ Unpaired Student's $t$-test $(\mathbf{D})$ One-way ANOVA with Dunnett's multiple comparison post-test. ns, not significant; ${ }^{*} p<0.05 ;{ }^{* *} p<0.01 ;{ }^{* * *} p<0.001 ;{ }^{* * *} p<0.0001$.

lysed. Consistent with previous findings ${ }^{16}$, SLC7A11 was strongly upregulated upon $\mathrm{H}_{2} \mathrm{O}_{2}$ treatment in $\mathrm{H} 1299$ p5 $3^{\text {null }}$ cells $(p<0.001)$ but not in $\mathrm{p} 53^{\mathrm{R} 273 \mathrm{H}}$ cells $($ Fig. $1 \mathrm{C})$. In a complementary approach, the effect of depleting NRF2 on TKT expression was assessed using siRNA to genetically knock down NRF2. TKT mRNA expression was found to be unchanged in response to NRF2 knockdown (Fig. 1D, Supplementary Fig. 1B). In contrast, SLC7A11 expression was downregulated upon NRF2 knockdown in p53 null cells but not p53 ${ }^{\mathrm{R} 273 \mathrm{H}} \mathrm{H} 1299$ cells, consistent with previous findings ${ }^{16}$. Taken together, these results suggest that TKT expression is not affected by mut-p53 regulation of NRF2 transcriptional activity in H1299 cells.

Transient knockdown of $T K T$ with siRNA slows cell growth and induces cell cycle arrest in H1299 cells independent of mutant-p53 expression. Having established that p53 status and NRF2 activation had no apparent bearing on TKT expression, we sought to uncover whether cell dependence on TKT varied with respect to p53 status. To examine this, TKT was transiently knocked down in H1299 and JHEso-Ad1 cells with the use of siRNA. Despite a highly efficient and rapid knockdown of TKT at the mRNA level across all cell lines (Fig. 2A), TKT protein appeared remarkably stable and did not noticeably decline until $96 \mathrm{~h}$ after transfection with siRNA (Fig. 2B). This rapid mRNA knockdown with lagging decrease in protein levels was common to all tested cell lines. Depletion of TKT reduced cell proliferation in H1299 cells but not JHEso-Ad1, irrespective of p53 status (Fig. 2C; Supplementary Fig. 2A,B). This diminished cell growth in $\mathrm{H} 1299$ cells could be completely rescued by the addition of N-acetyl-cysteine (NAC), a potent antioxidant (Fig. 2D), suggesting that the impact of TKT on cellular redox balance may be tantamount to its effect on cell growth. Because maintaining an optimal redox state is necessary for proper cell replication ${ }^{30}$, the impact of TKT on cell cycle progression with respect to p53 status was also investigated. Knockdown of TKT induced arrest in the G0/G1 phase in H1299 cells (Fig. 2E; Supplementary Fig. 2C-F). Moreover, this effect was seen in both $\mathrm{H} 1299$ p53 $3^{\text {null }}$ and p53 ${ }^{\mathrm{R} 273 \mathrm{H}}$ cell lines. Altogether, these data support the notion that the role of TKT in moderating oxidative stress may be required for cell proliferation, p53 status notwithstanding, but is cell context dependent. 
Transient knockdown of $T K T$ with siRNA sensitises p53 ${ }^{\text {null }}$ cells to APR-246. We next investigated the relationship between TKT and the antioxidant-depleting drug APR-246. Analysis of the NCI-60 human tumour cell line screen found that TKT expression correlated with sensitivity to PRIMA-1, an unmethyl-

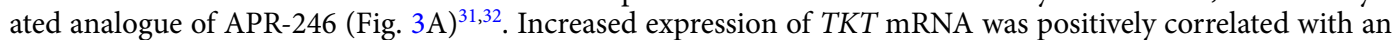
increased growth inhibitory concentration (GI50) of APR-246. This suggests a potential protective effect of TKT against APR-246.

Transient knockdown of TKT did not alter the response of cells expressing mutant p53 protein, nor when the endogenous mutant TP53 was knocked out in JH-EsoAd1 (Supplementary Fig. 3A-E). Interestingly, we noticed that knockdown of TKT increased sensitivity of H1299 p53 null cells to low doses of APR-246, with a coefficient of interaction (CI) of $0.66 \pm 0.06$ indicative of synergistic activity (Fig. 3B-D, Supplementary Fig. 3F). Synergistic interactions were also observed in Cas9-expressing $\mathrm{H} 1299$ p $53^{\text {null }}$ cells transduced with two independent synthetic guide RNAs (sgRNAs) targeting TKT (Fig. 3E, Supplementary Fig. 3F). To investigate this further, we utilised another cell line with an endogenous TP53 mutation that results in a p53 null status (OACP4C, Fig. 3F) and HCT116 cells (Fig. 3G), which express wild-type p53 protein. Similar to H1299 p53 ${ }^{\text {null }}$ cells, OACP4C p53 ${ }^{\text {null }}$ cells showed synergistic activity between TKT knockdown and APR-246 (Supplementary Fig. 3A), as well as a significant decrease in the GI50 of APR-246 in TKT depleted cells. In contrast, the maximal CI in HCT116 cells was $0.78 \pm 0.12$ (Supplementary Fig. $3 \mathrm{~A}$ ) indicative of an additive/borderline-synergistic effect. Thus, knockdown of TKT is synergistic with APR-246 in cancer cell lines with an endogenous p53 null phenotype.

Transient knockdown of TKT augments oxidative stress levels, resulting in increased cell death. We next investigated whether the sensitisation of H1299 p53 $3^{\text {null }}$ cells to APR-246 through TKT knockdown was a product of the combined effect of these conditions on oxidative stress levels. Treatment with APR-246 $(25 \mu \mathrm{M})$ for a relatively short period $(18 \mathrm{~h})$ did not significantly alter the levels of NADPH (Fig. 4A) nor mitochondrial superoxides in H1299 cells (Fig. 4B; Supplementary Fig. 3G). In contrast, TKT knockdown significantly decreased NADPH levels (Fig. 4A) and increased mitochondrial ROS in H1299 p53 ${ }^{\text {null }}$ cells, which was further augmented by APR-246 $(p<0.05)$ (Fig. 4B; Supplementary Fig. 3G). Treatment with NAC entirely rescued the combination effect of TKT knockdown and APR-246 on cell viability $(p<0.001)$ (Fig. 4C). Altogether, these results indicate that the combination of TKT knockdown and APR-246 treatment induces a greater oxidative stress environment than either condition alone. The fact that the sensitisation of H1299 p53 $3^{\text {null }}$ cells to APR-246 by TKT knockdown was rescued by antioxidant treatment suggests that greater intracellular ROS levels are the cause of the increased cell death. However, transient knockdown of TKT did not sensitise H1299 cells to Erastin, a known inducer of oxidative stress (Fig. 4D, Supplementary Fig. 3I), suggesting a more complex mechanism at play in the context of APR-246.

Overexpression of $T K T$ reduces sensitivity to APR-246 in H1299 p53 ${ }^{\text {null }}$ cells. We next determined the effect of overexpressing TKT on response to APR-246 in H1299 cells. Consistent with the effect of transient TKT knockdown, overexpression of TKT (Fig. 5A,B) conferred resistance to APR-246 in H1299 p53 ${ }^{\text {null }}$ cells (Fig. 5C,D; Supplementary Fig. 4A) but this effect was not seen in H1299 p53 ${ }^{\mathrm{R} 273 \mathrm{H}}$ (Supplementary Fig. 4B) or p53 ${ }^{\text {Ri75H }}$ cells (Supplementary Fig. 4C). Consistent with this, overexpression of TKT significantly reduced basal mitochondrial ROS and protected against APR-246-induced superoxides in H1299 p53 ${ }^{\text {null }}$ cells (Fig. 5E; Supplementary Fig. 4D). However, the protective effect of TKT overexpression in these cells did not extend to long-term survival as assessed in clonogenic assays (Supplementary Fig. 4E). Combined, these data support the notion that overexpression of TKT delays the onset of APR-246-induced cell death in H1299 p53 $3^{\text {null }}$ cells.

Inhibition of NADPH production slows cell growth in $\mathrm{H} 1299 \mathrm{p} 53^{\text {null }}$ cells but does not affect sensitivity to APR-246 or induce cell cycle arrest. A major role of TKT in the PPP is the shuttling of intermediates back into glycolysis, from where they return to the oxidative arm of the PPP and contribute to the production of $\mathrm{NADPH}^{33}$. Given this, we next investigated whether treatment with 6-aminonicotinamide (6AN), an inhibitor of NADPH production (Supplementary Fig. 5A), would elicit the same effects as TKT knockdown. 6AN treatment elicited a significantly greater reduction in growth rate than that caused by TKT knockdown, but this effect was not enhanced when TKT knockdown and 6AN treatment were administered concomitantly (Fig. 6A). Further, 6AN treatment did not sensitise H1299 p53 $3^{\text {null }}$ cells to APR-246 (Fig. 6B), and no significant shift in the GI50 of APR-246 was observed in these cells $(p=0.14)$, despite significantly increased mitochondrial superoxides following treatment with 6AN, indicating drug activity (Fig. 6C; Supplementary Fig. 5B). The impact of 6AN on cell cycle progression was also assessed. After $24 \mathrm{~h}$ of treatment with 6AN, neither H1299 p5 $3^{\text {null }}$ nor p53 $3^{\text {R273H }}$ cells showed any sign of cell cycle arrest (Fig. 6D; Supplementary Fig. 5C-F). This is in stark contrast to the clear interference with cell cycling caused by TKT knockdown. Taken together, these results suggest some similarities between the consequences of TKT knockdown and inhibition of NADPH production by $6 \mathrm{AN}$, but $6 \mathrm{AN}$ treatment does not entirely phenocopy the effects of TKT gene knockdown.

\section{Discussion}

Despite the clear parallels between these factors relating to antioxidant response mechanisms, the relationship between mut-p53 and the role of the PPP and TKT in the antioxidant response remains poorly understood. This work aimed to delineate the relationship between TKT and mut-p53, as well as TKT and the drug APR-246, which has both mutant-p53 reactivating and oxidative stress-inducing mechanisms. Using isogenic models of p $53^{\text {null }}$ or mut-p53 cells and additional cell lines with varying p53 status, we examined the effects of perturbations to TKT on oxidative stress and sensitivity to APR-246, relative to p53 status. Our findings indicate a potential role for TKT in modulating sensitivity to APR-246, but suggest that this is likely not via altering redox balance. 
A
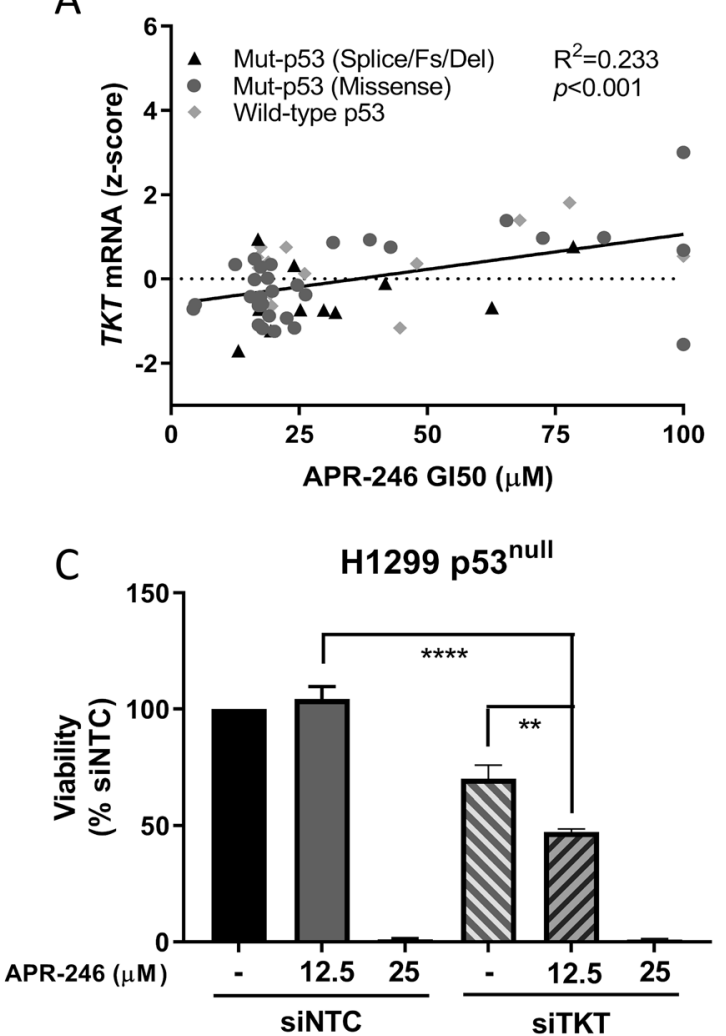

B
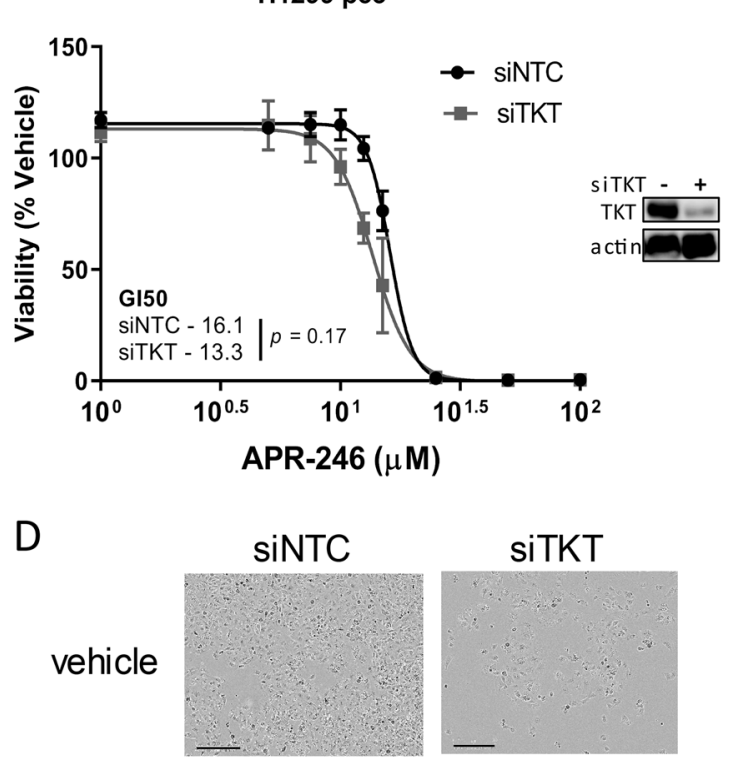

APR-246

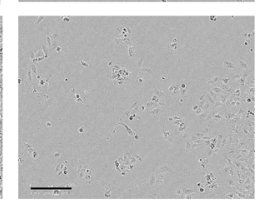

$\mathrm{E}$

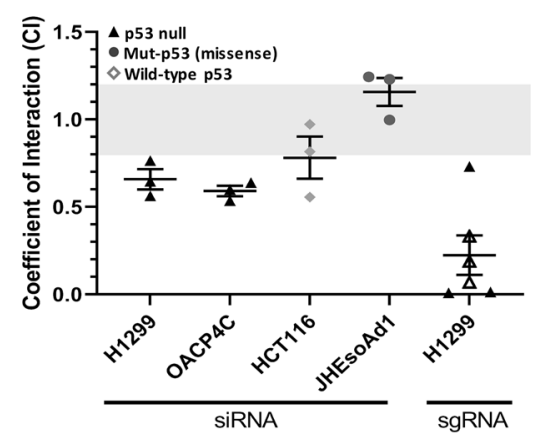

$\mathrm{F}$
OACP4C

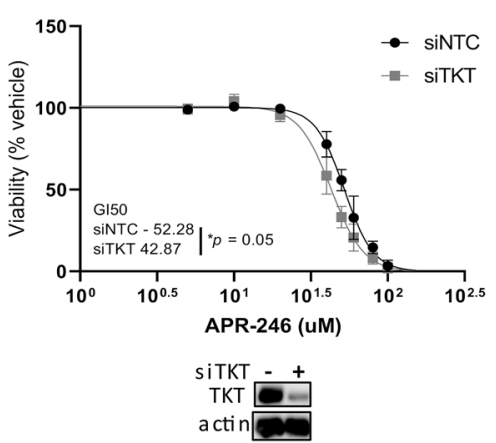

G

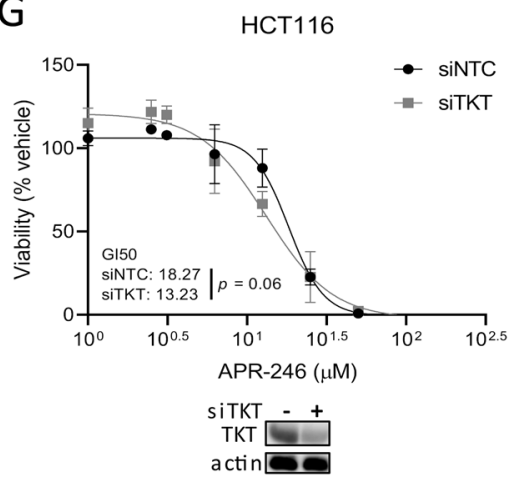

Figure 3. Acute knockdown of $T K T$ sensitises $p 53^{\text {null }}$ cells to APR-246. (A) Correlation between TKT mRNA levels and APR-246 GI50 (50\% growth inhibitory concentration) across the NCI-60 cancer cell line panel ${ }^{31}$. Each point represents an individual cell line. The NCI-60 is a panel of 60 diverse human cancer cell lines used by the National Cancer Institute for therapeutic development ${ }^{32}$. (B) (left) Cell viability (AlamarBlue) in H1299 cells following $72 \mathrm{~h}$ treatment with increasing concentrations of APR-246, administered $24 \mathrm{~h}$ after TKT knockdown with siRNA, normalised to vehicle control (siNTC, non-targeting control; siTKT, TKT siRNA (right) Western blots for TKT in H1299 cells following TKT knockdown with siRNA for $96 \mathrm{~h}$, showing actin as a loading control. (C) Cell viability (AlamarBlue) in H1299 parental (p53 $3^{\text {null }}$ ) cells following $72 \mathrm{~h} \mathrm{APR-246}$ treatment (12.5 or $25 \mu \mathrm{M}$ ), administered after $24 \mathrm{~h}$ TKT knockdown with siRNA, normalised to siNTC vehicle control. (D) Digital microscope images (Incucyte FLR) depicting cell death following $72 \mathrm{~h}$ treatment with APR-246 $(15 \mu \mathrm{M}$ ), administered $24 \mathrm{~h}$ after TKT knockdown with siRNA (scale bars $=100 \mu \mathrm{m}$ ). (E) Interaction between TKT knockdown and APR-246 in H1299, OACP4C, HCT116 and JH-EsoAd1 cells based on the Bliss Independence model. Data represent most synergistic interaction across APR-246 dose response. A coefficient of interaction $<0.8$ indicates synergy, $0.8-1.2$ indicates additivity and $>1.2$ indicates antagonism. Cell viability (AlamarBlue) in (F) OACP4C and (G) HCT116 cells following $72 \mathrm{~h}$ treatment with increasing concentrations of APR-246, administered $24 \mathrm{~h}$ after TKT knockdown with siRNA, normalised to vehicle control (bottom) Western blots for TKT following $96 \mathrm{~h}$ knockdown with siTKT, showing actin as a loading control (siNTC, nontargeting control; siTKT, TKT siRNA. Data represent mean, error bars $=$ SEM, $\mathrm{n}=3$ for all studies. (C) One-way ANOVA with Dunnett's multiple comparison post-test. ns, not significant; ${ }^{*} p<0.05 ;{ }^{* *} p<0.01$; ${ }^{* *} p<0.001$; $* * * * p<0.0001$. 
A
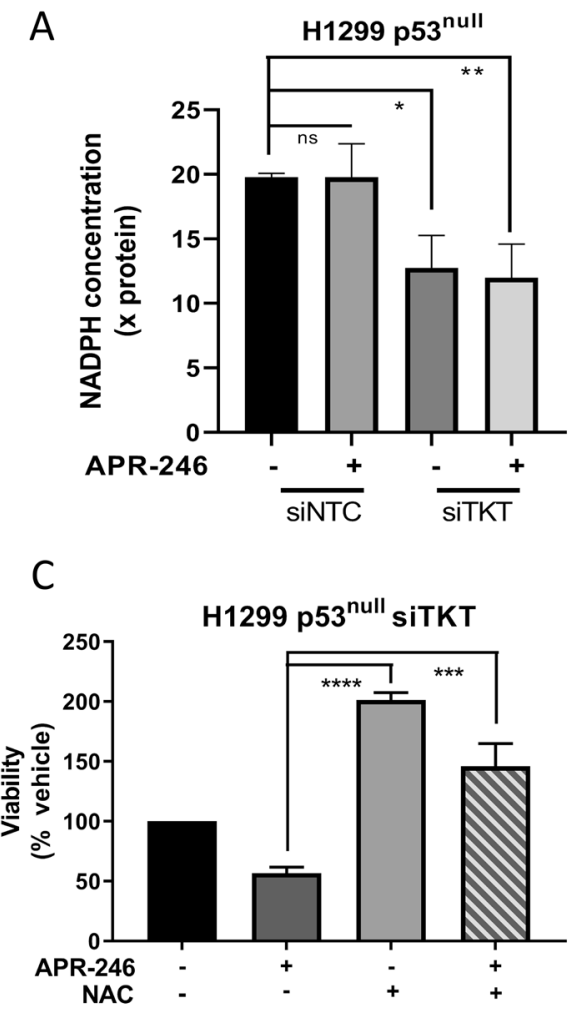

B

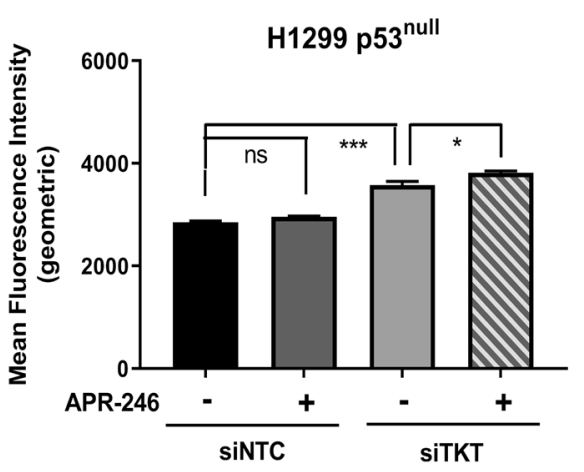

D

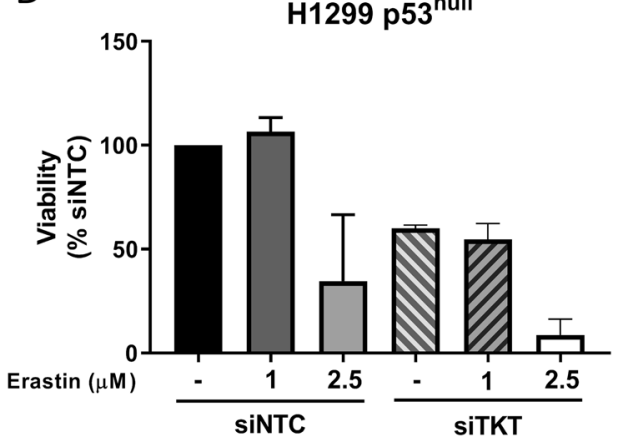

Figure 4. Transient knockdown of TKT with siRNA augments oxidative stress levels, but does not increase sensitivity to Erastin in H1299 p53 ${ }^{\text {null }}$ cells. (A) Concentration of NADPH, normalised to total protein concentration, in H1299 parental (p53 ${ }^{\text {null }}$ ) cells following $18 \mathrm{~h}$ APR-246 $(25 \mu \mathrm{M})$ treatment administered after $72 \mathrm{~h}$ TKT knockdown with siRNA (siNTC, non-targeting control; siTKT, TKT siRNA). (B) Geometric mean fluorescence intensity of MitoSOX Red in H1299 parental (p53 ${ }^{\text {null }}$ ) cells following 18 h APR-246 (25 $\mu$ M) treatment administered after $72 \mathrm{~h} T K T$ knockdown with siRNA, measured with flow cytometry (siNTC, non-targeting control; siTKT, TKT siRNA). (C) Cell viability in H1299 parental (p53 $3^{\text {null }}$ ) cells following $72 \mathrm{~h}$ APR-246 $(15 \mu \mathrm{M})$ treatment and/or $\mathrm{N}$-acetyl-cysteine (NAC, $5 \mathrm{mM}$ ) supplementation, following $24 \mathrm{~h}$ TKT knockdown with siRNA, shown as a percentage of vehicle control. (D) Cell viability (AlamarBlue) in H1299 cells following $72 \mathrm{~h}$ treatment with 1 or $2.5 \mu \mathrm{M}$ of Erastin, administered $24 \mathrm{~h}$ after TKT knockdown with siRNA, normalised to siNTC vehicle control (siNTC, non-targeting control; siTKT, TKT siRNA). Data represent mean, error bars $=\mathrm{SEM}, \mathrm{n}=3$ for all studies. $(\mathbf{A}, \mathbf{B}, \mathbf{C})$ One-way ANOVA with Dunnett's multiple comparison post-test. ns, not significant; ${ }^{\star} p<0.05 ;{ }^{* *} p<0.01 ;{ }^{* * *} p<0.001 ;{ }^{* * *} p<0.0001$.

Previous studies dictate that accumulated mut-p53 inhibits the transcriptional ability of NRF2 ${ }^{16,19,20}$ whilst NRF2 has been found to transcriptionally activate $T K T^{25}$. From this, it may be inferred that through inhibition of NRF2, accumulated mut-p53 may impact transcription of TKT. In contrast, our work determined that neither manipulation of mut-p53 nor NRF2 had any bearing on expression of TKT in H1299 or JH-EsoAd1 cells (Fig. 1A-D). This suggests that mechanisms of TKT regulation may differ among cancer cell types or those with altered dependency on NRF2. For example, TKT was found to be a transcriptional target of NRF2 in A549 cells which harbour endogenous KEAP1 mutation, leading to constitutively active NRF2 in these cells ${ }^{26}$. Given that neither cell line used in this study contain KEAP1 mutations, is it possible that this difference may account for the discrepancy between our work and previous findings.

Consistent with previous findings ${ }^{25}$, we found that TKT depletion hinders cell growth by arresting the cell cycle, and that this occurred irrespective of p53 status (Fig. 2C,E). That this could be rescued with antioxidant treatment (i. e. NAC; Fig. 2D) is in congruence with previous studies involving TKT and other PPP enzymes, such as ribose-5-phosphate isomerase (RPIA) ${ }^{34}$. RPIA converts ribulose-5-phosphate (Ru5P) into ribose-5-phosphate $(\mathrm{R} 5 \mathrm{P})$ in the PPP and thus plays a major role in ribonucleotide biosynthesis ${ }^{35}$. This similarity strongly suggests an ability of NAC to counteract the decline in ribonucleotide synthesis that is likely caused by depletion of these PPP enzymes.

Although these early data did not identify any relationship between TKT and mut-p53, it remains that, according to NCI-60 data, a correlation exists between TKT expression and sensitivity to PRIMA-1 (Fig. 3A). Critically, we found that transient knockdown or knockout of TKT sensitised only p53 ${ }^{\text {null }}$ cells, and not cells expressing mut-p53, to low doses of APR-246 (Fig. 3B-F, Supplementary Fig. 3A-F), whilst overexpression of TKT conferred resistance to the drug, again only in p53 null cells (Fig. 5C). Notably, in contrast to cells with an endogenous p53 null mutation, TKT knockdown did not increase sensitivity in JH-EsoAd1 cells in which the 
A

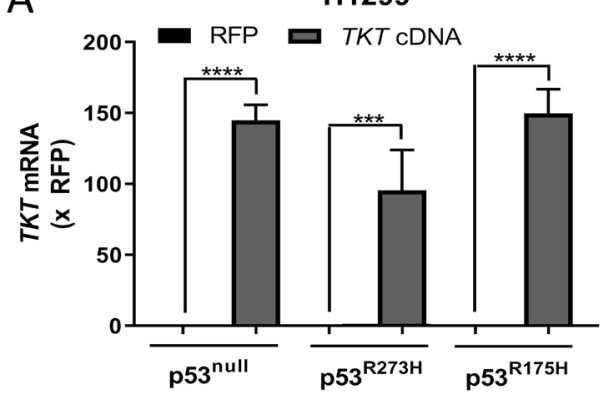

B

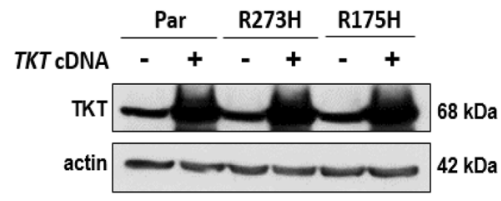

C

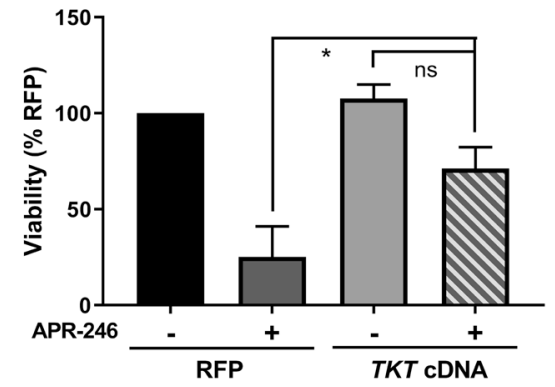

D

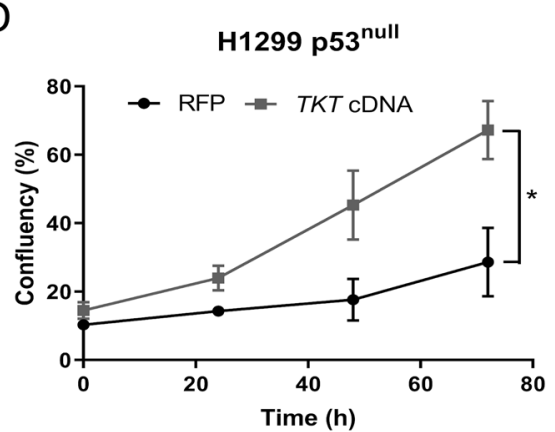

$\mathrm{E}$

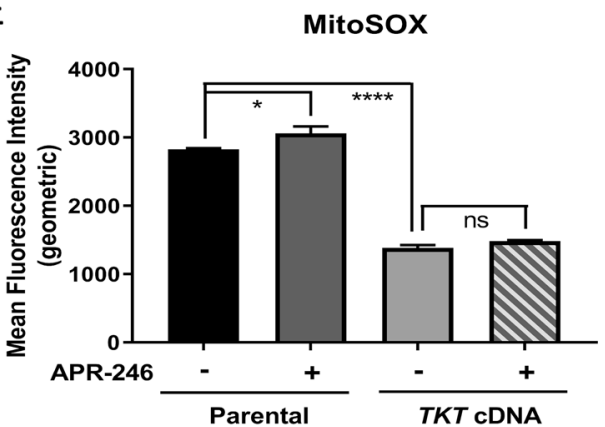

Figure 5. Overexpression of TKT induces resistance to APR-246 in p53-null cells and protects against APR-246-induced superoxides. (A) TKT mRNA expression and (B) western blots for TKT in H1299 parental $\left(\mathrm{p} 53^{\text {null }}\right), \mathrm{p} 53^{\mathrm{R} 273 \mathrm{H}}$ and $\mathrm{p} 53^{\mathrm{R} 175 \mathrm{H}}$ cells with either TKT or red fluorescent protein (RFP) overexpression. (A) Expression normalised to RFP, (B) actin is shown as loading control. (C) Cell viability and (D) proliferation of H1299 parental (p53 ${ }^{\text {null }}$ ) cells with either TKT or red fluorescent protein (RFP) overexpression following $72 \mathrm{~h}$ APR-246 treatment $(15 \mu \mathrm{M})$. (E) Geometric mean fluorescence intensity of MitoSOX Red in H1299 parental (p53 ${ }^{\text {null }}$ ) cells either untransfected (Parental) or overexpressing TKT (TKT cDNA) with $18 \mathrm{~h}$ APR-246 treatment $(25 \mu \mathrm{M})$ measured with flow cytometry. Data represent mean, error bars $=$ SEM, $\mathrm{n}=3$ for all studies excluding (B) where blots are representative of 2 independent experiments. (A) Unpaired Student's $t$-test on raw data. (C,E) One-way ANOVA with Dunnett's multiple comparison post-test. (D) Unpaired Student's $t$-test. ns, not significant; ${ }^{\star} p<0.05,{ }^{* *} p<0.001,{ }^{* * *} p<0.0001$.

endogenous missense mut-p53 had been knocked out using CRISPR/Cas9. While the reason for this remains unclear, we speculate that this may reflect differences between tumour cells that have evolved without the ability to express any $\mathrm{p} 53$ protein (H1299 and OACP4C cells) compared to cells that evolved expressing a missense mut-p53 that was then knocked out.

Our results in mut-p53 expressing cells were surprising, given previous findings that mut-p53 expressing cells are highly sensitive to further assaults on cellular redox balance (particularly the antioxidant-depleting effects of APR-246) due to inherent impaired antioxidant function ${ }^{11,16}$. However, mut-p53 has been shown to transactivate a range of anti-apoptotic and growth enhancing factors ${ }^{8,9,36,37}$ as well as alter tumour cell metabolism to promote survival ${ }^{7,38,39}$. In fact, it has been found that the antioxidant thioredoxin is upregulated in mut-p53 expressing cells ${ }^{11}$, which alludes to a thioredoxin-dependent mechanism that allows mut- 53 cells to compensate for APR-246-induced glutathione depletion. As such, it is possible that the mut-p53 expressing cells were unaffected by the consequences of TKT knockdown due to upregulation of pro-survival measures or prioritisation of alternative metabolic pathways.

Upon further investigation of oxidative balance, and consistent with the findings of Xu et al. ${ }^{25}$, we found that in all cell types, knockdown (Fig. 4A,B) and overexpression (Fig. 5E) of TKT increased and decreased intracellular ROS burden, respectively. These data suggest that modulations to TKT influence flux through the oxidative arm of the PPP and subsequent production of NADPH. Intriguingly, this occurred in both p53 $3^{\text {null }}$ and mut-p53 expressing cells, leading to the conundrum of why TKT depletion only induced sensitivity to APR- 246 in p53 $3^{\text {null }}$ cells, whilst the effect on ROS levels was also seen in mut-p53 expressing cells. Cancer cells natively exhibit higher levels of oxidative stress than untransformed cells ${ }^{30,40,41}$. Basal ROS levels in mut-p53 expressing cells were found to be higher still, in comparison to $53^{\text {null }}$ cells (Supplementary Fig. $3 \mathrm{H}$; compare with Fig. $4 \mathrm{~B}$ ), congruent with previous findings ${ }^{16,21}$, likely due to the suppressing effect of mut-p53 on NRF2-dependent antioxidant pathways. Despite higher levels of oxidative stress, mut-p53 cancers have been found to show improved survival and augmented proliferation in response to oxidative stress ${ }^{21}$. Mut-p53 is also thought to be able to commandeer additional pro-survival pathways ${ }^{8,9,36,37}$. Thus, it is possible that due to these gains-of-function, mut-p53 expressing cells were able to escape the effects of TKT knockdown that increased APR-246-induced cell death in p53 ${ }^{\text {null }}$ cells. 
A

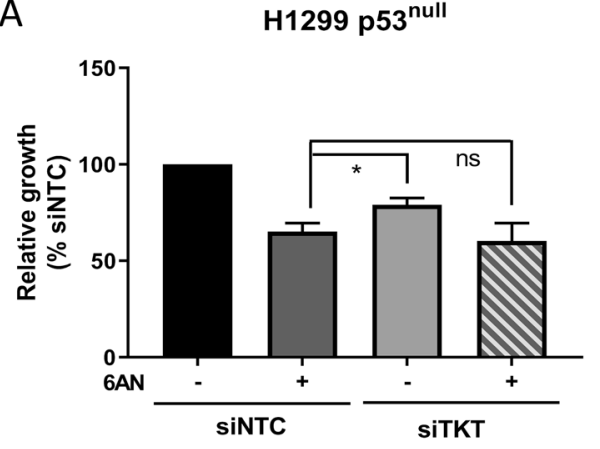

C

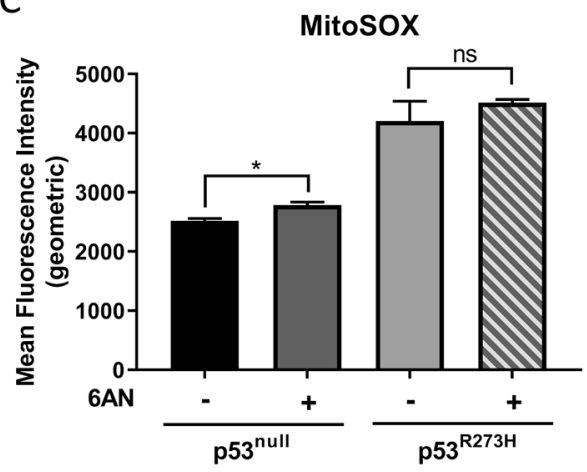

B

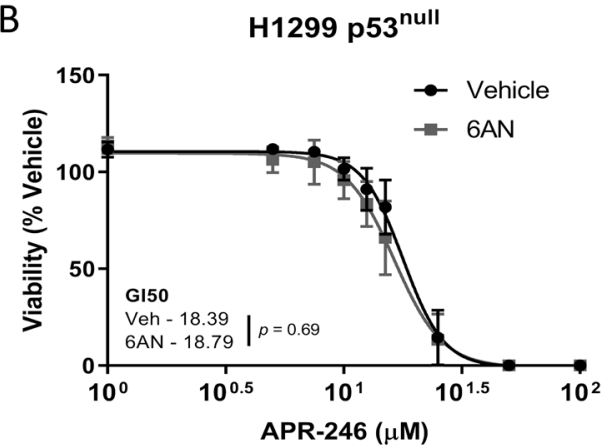

D

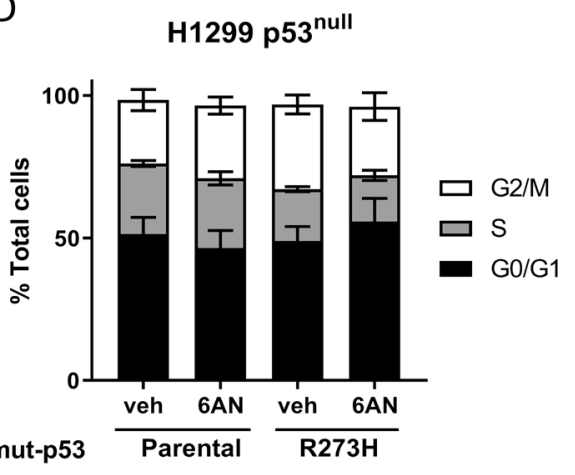

Figure 6. Inhibition of NADPH production slows cell growth but does not sensitise p53 $3^{\text {null }}$ cells to APR-246 or induce cell cycle arres. (A) Cell viability in H1299 parental ( $\mathrm{p} 53^{\text {null }}$ ) cells following TKT knockdown with siRNA or transfection with non-targeting control in combination with $72 \mathrm{~h}$ 6-aminonicotinamide $(6 \mathrm{AN} ; 10 \mu \mathrm{M})$ treatment, normalised to non-targeting control (siNTC, non-targeting control; siTKT, TKT knockdown with siRNA). (B) Cell viability in H1299 parental (p53 $\left.3^{\text {null }}\right)$ cells following $72 \mathrm{~h} 6 \mathrm{AN}(10 \mu \mathrm{M})$ or vehicle treatment in combination with increasing doses of APR-246. (C) Geometric mean fluorescence of MitoSOX Red in H1299 parental ( $\left.\mathrm{p} 53^{\text {null }}\right)$ and $\mathrm{p} 53^{\mathrm{R} 273 \mathrm{H}}$ cells following $72 \mathrm{~h}$ treatment with $6 \mathrm{AN}(10 \mu \mathrm{M})$ or vehicle measured by flow cytometry. (D) Changes in cell cycle distribution in (left) H1299 parental (p53 ${ }^{\text {null }}$ ) and (right) p53 ${ }^{\mathrm{R} 273 \mathrm{H}}$ cells following $24 \mathrm{~h}$ treatment with $6 \mathrm{AN}$ (veh, vehicle control). Data represent mean, error bars $=\mathrm{SEM}, \mathrm{n}=3$ for all studies. (A) One-way ANOVA with Dunnett's multiple comparison post-test. (C) Unpaired Student's t-test. ns, not significant; ${ }^{*} p<0.05$.

TKT has a dualistic role in the PPP and its activity modulates depending on the metabolic needs of the cell ${ }^{33}$. It accomplishes this by either shuttling intermediates back towards the oxidative arm of the PPP to produce NADPH in response to oxidative demand, or producing R5P to promote production of ribonucleotides ${ }^{33}$. In order to separate these effects and their potential role in altering sensitivity to APR-246, 6-aminonicotinamide (6AN), a compound that impedes NADPH production but is not known to effect production of ribonucleotides, was utilised. 6AN inhibits the two major NADPH-producers in the PPP, 6-phosphogluconoate (6PGD) and glucose-6-phosphate dehydrogenase (G6PD ${ }^{42,43}$. In this way, the isolated effect of NADPH depletion on APR-246 sensitivity was able to be investigated, without the alternative function of TKT (i. e. ribonucleotide production) confounding the results. Intriguingly, 6AN slowed cell growth (Fig. 6A) despite eliciting no effect on cell cycle progression (Fig. 6D), irrespective of p53 status. In contrast to transient TKT knockdown, 6AN treatment did not significantly alter sensitivity to APR-246 in H1299 p53 ${ }^{\text {null }}$ cells (Fig. 6B), despite eliciting a similar effect on intracellular ROS levels (Fig. 6C). This suggests that impaired R5P production may be the driving force behind TKT knockdown-induced APR-246 sensitivity in p5 $3^{\text {null }}$ cells, rather than increased levels of ROS due to inadequate antioxidant mechanisms. Additionally, TKT knockdown did not increase sensitivity to erastin, a known oxidative stress inducer, indicating that loss of TKT expression is not broadly sensitising cells to oxidative stressmediated cell death. Imperatively, this suggests that the mechanism of action of APR-246 is more complex than previously described and may incorporate aspects of macromolecule biosynthesis that have not been explored previously in this context. Future work is therefore required to examine the effect of TKT knockdown on R5P production and the impact of this on APR-246 sensitivity and how this is related to p53 status.

Despite similar TKT expression levels across cells in this model with varying p53 status, a distinct change in sensitivity to APR-246 was observed when TKT was ectopically overexpressed or transiently depleted in p $53^{\text {null }}$ cells. This, combined with correlative data from the NCI-60 human tumour cell line screen (Fig. 3A), suggests that TKT expression regulates sensitivity to APR-246, at least in $\mathrm{p} 53^{\text {null }}$ tumours. Of particular interest is the evidence that sensitivity of mut-p53 cells to APR-246 is not regulated in this same way in this model. We propose a model to explain this observation based on the induction of wild-type-p53-like function in mut-p53 cells when treated with APR-246 (Supplementary Fig. 6). Wild-type-p53 has been found to inhibit activity of G6PD, the 
rate-limiting enzyme of the PPP, upstream of TKT, by preventing dimerization of the enzyme ${ }^{44}$. Because treatment with APR-246 induces wild-type-p53-like conformation and activity in mut-p53 cells, mut-p53 cells treated with APR-246 sustain impeded function of the PPP via G6PD inhibition. Therefore, due to upstream interference and consequential limited flux through the PPP, modulations of downstream TKT may have no effect on APR246 activity. Treatment of p53 null cells with APR-246 would not confer this same wild-type-p53-like effect. Thus, modulations of TKT in $\mathrm{p} 53^{\text {null }}$ cells would strongly interfere with the previously intact PPP, rendering the cells more susceptible to APR-246 treatment. This is consistent with our finding that upstream interference with the PPP via G6PD inhibition by $6 \mathrm{AN}$ had no synergistic or additive effect in combination with TKT modification in any cell lines, irrespective of p53 status. In summary, this study bridges the gaps in current knowledge surrounding the relationship between the PPP, mut-p53 cancers and therapies. The findings indicate that whilst mut-p53 is known to interfere with NRF2, it did not affect TKT in the investigated models. Furthermore, it is proposed that regulation of TKT is not as simple as per the previously suggested mechanism of NRF2 activation ${ }^{25}$, and that other factors must certainly be at play. This study also addressed the growing complexity of mut-p53 gain-offunction capabilities. Given the failure of NRF2 modulation to affect TKT expression in this study, future research is needed to explore the role of mut-p53 in regulating the antioxidant response and its influence on the PPP. The present findings also mar the current predilection in research for targeting cellular redox balance as an avenue for chemotherapeutics, particularly for mut-p53 cancers. This is due, in part, to growing evidence of the complex nature of mut-p53 in the antioxidant response, as well as the uncovering of the potential influence of external factors. Finally, this study has identified TKT as a determinant for APR-246 sensitivity in p53 ${ }^{\text {null }}$ cells, which may open a new avenue for exploration and potentially allow for personalised, effective treatment for $\mathrm{p} 53^{\text {null }}$ cancers.

\section{Materials and methods}

Cell culture. HEK293T (RRID:CVCL_0063) cells were purchased from American Tissue Culture Collection. NCI-H1299 (RRID:CVCL_0060) and mutant p53 expressing sublines were a kind gift from Prof. Ygal Haupt (Peter MacCallum Cancer Centre, Melbourne, Australia). JH-EsoAd1 (RRID:CVCL_8098) cells were obtained from Professor James Eshleman (Johns Hopkins University, Baltimore, Maryland, USA). OACP4C (RRID:CVCL_1843) cells were obtained from Rebecca Fitzgerald (University of Cambridge, UK). HCT116 (RRID:CVCL_0291) cells were obtained from Karen Sheppard (Peter MacCallum Cancer Centre, Australia). H1299, JH-EsoAd1 and OACP4C cells were cultured in RPMI 1640 containing 2.5 mM L-glutamine (Gibco, ref 11875-093), HCT116 and HEK293T cells were cultured in DMEM (Gibco, ref 11965-092) and all media supplemented with $10 \%$ heat inactivated foetal bovine serum (FBS) and $1 \%$ penicillin and streptomycin (Gibco, ref 15140-122) unless otherwise stated. All cells were incubated at $37^{\circ} \mathrm{C}$ and $5 \% \mathrm{CO}_{2}$, STR genotyped to confirm identity and regularly tested for mycoplasma contamination. Unless otherwise indicated, seeding densities were as follows for H1299 and JH-EsoAd1 cells: $2 \times 10^{3}$ in 96 -well format, $4 \times 10^{5}$ for less than $24 \mathrm{~h}$ incubation and $2 \times 10^{5}$ for incubation times between 24 and $48 \mathrm{~h}$ in 6 -well format and $6 \mathrm{~cm}$ dishes. OACP4C cells were seeded at $1 \times 10^{4}$ and HCT116 at $3 \times 10^{3}$ in 96 -well format.

Transient TKT and NRF2 knockdown. Cells were reverse transfected with $40 \mathrm{nM}$ NRF2 (siGenome Smartpool, Dharmacon), TKT or non-targeting control siRNA pools (ON-TARGETplus SMARTpool, Dharmacon) using Lipofectamine RNAiMax solution (Life Technologies) as per manufacturer's guidelines. Knockdown efficiency was assessed by reverse transcription quantitative polymerase chain reaction (RT-qPCR) and western blotting. siRNA sequences are detailed in Supplementary Table 1.

TKT knockout using CRISPR/Cas9. H1299 cells expressing constitutive Cas9 endonuclease (FUCas9Cherry, kind gift of Marco Herold, WEHI, Australia) were reverse transfected with two independent TKT sgRNA (Integrated DNA Technologies, IDT) at $30 \mathrm{nM}$ using Lipofectamine RNAiMax solution (Life Technologies) according to the manufacturer's protocol. Knockout efficiency was assessed by western blotting.

Overexpression ofTKT. TKT was ectopically overexpressed in H1299 cells using Precision LentiORF TKT with stop codon (clone ID: PLOHS_100005708) or red fluorescent protein (RFP) as a control. Viral particles were produced by transfecting HEK293T cells using the Lenti-X Packaging System (Clontech Laboratories) as per manufacturer's directions. Subsequently, target cell lines were transduced with viral supernatants in the presence of $8 \mathrm{ng} / \mu \mathrm{L}$ polybrene. Transduced cells were collected via cytometric sorting (BD Fusion3, BD Bioscience). Expression was confirmed by RT-qPCR and western blotting.

Gene expression using RT-qPCR. Cells were seeded in $6 \mathrm{~cm}$ dishes and allowed to adhere overnight. Following the relevant experiments, extraction and purification of RNA was conducted using Nucleospin RNA kit (Macherey-Nagel) before reverse transcription to cDNA using Transcriptor First Strand cDNA Synthesis Kit (Roche) as per manufacturer's instructions, including the optional denaturing step. Quantitative PCR (qPCR) was conducted using LightCycler 480 SYBR-Green qPCR (Roche) as per manufacturer's protocol, with gene expression normalised within each sample to GAPDH and analysed using the $\Delta \Delta \mathrm{Ct}$ method. Primer sequences were obtained using the NCBI Primer Blast application and are detailed in Supplementary Table 2. Product sizes were confirmed via electrophoresis on $1 \%$ agarose gels with size comparison to Hyperladder 100 bp (Bioline).

Western blotting. Cells were seeded in $6 \mathrm{~cm}$ dishes and allowed to adhere overnight. Following the relevant experiments, cells were lysed on ice in RIPA buffer ( $1 \mathrm{mM}$ EDTA; $1 \% \mathrm{v} / \mathrm{v}$ NP40; 0.5\% w/v sodium deoxycholate; $0.1 \% \mathrm{v} / \mathrm{v}$ SDS; $50 \mathrm{mM}$ sodium fluoride; $1 \mathrm{mM}$ sodium pyrophosphate in PBS, SigmaAldrich) containing 
phosphatase (PhosphoSTOP, Roche) and protease (Complete ULTRA, Roche) inhibitors. Protein concentrations were quantified using the DC Protein assay (Bio-Rad). Equivalent amounts of protein lysates were boiled, resolved by SDS-PAGE using 10\% w/v acrylamide gels, and transferred to polyvinylidene difluoride membranes. Membranes were incubated for $1 \mathrm{~h}$ in blocking buffer (5\% w/v skim milk, 0.05\% v/v Tween-20 in Tris-buffered saline; TBS-T) and probed overnight at $4{ }^{\circ} \mathrm{C}$ with the primary antibody. Blots were washed thrice in wash buffer $(0.05 \% \mathrm{v} / \mathrm{v}$ TBS-T) for $10 \mathrm{~min}$, followed by incubation with peroxidase-conjugated secondary antibody (Dako) for $1 \mathrm{~h}$ at room temperature. Proteins were visualised by Amersham ECL western Blotting Detection Reagents (GE Life Sciences) or ECL Plus western blotting substrate kit (Thermo Scientific). Anti- $\beta$-actin or anti-GAPDH antibodies were used to assess protein loading. Antibodies are detailed in Supplementary Table 3.

Cell cycle analysis. Cells were seeded in 24-well plates and exposed to siRNA TKT knockdown for $96 \mathrm{~h}$, 6 -aminonicotinamide (6AN) for $72 \mathrm{~h}$ or vehicle prior to harvesting. Cells were washed with PBS, trypsinised, pelleted by centrifugation at $2400 \mathrm{rpm}$ for $5 \mathrm{~min}$ and the supernatant removed before resuspension in PBS supplemented with $1 \% \mathrm{v} / \mathrm{v}$ FBS and fixing in ice cold $100 \%$ ethanol. Cells were then pelleted again and washed twice with $\mathrm{PBS} / 1 \% \mathrm{v} / \mathrm{v}$ FBS prior to staining for $90 \mathrm{~min}$ at room temperature in the dark with $25 \mu \mathrm{g} / \mathrm{mL}$ propidium iodide (Sigma-Aldrich) and $40 \mu \mathrm{g} / \mathrm{mL}$ RNAse A in PBS/1\% v/v FBS. At least $1 \times 10^{4}$ single cell events were recorded by flow cytometry (BD FACSCanto II, BD Bioscience) and analyzed using Flowlogic software (Inivai Technologies). The proportion of cells in each phase of the cell cycle was expressed as a percentage of total cell population.

NADPH measurement. Total NADPH was measured using a NADPH Assay Kit (Abcam) as per the manufacturer's instructions. Cells $\left(1 \times 10^{5}\right.$ per well) were seeded in 6-well plates and exposed to siRNA TKT knockdown for $96 \mathrm{~h}$ or $24 \mathrm{~h}$ before treatment with APR-246 $(25 \mu \mathrm{M})$ for $18 \mathrm{~h}$ or $6 \mathrm{AN}(10 \mu \mathrm{M})$ for $72 \mathrm{~h}$ respectively. Cells were washed with PBS, harvested by scraping in lysis buffer and centrifuged. Supernatant was extracted and absorbance at $460 \mathrm{~nm}$ was measured using a Cytation 3 microplate reader (Molecular Devices). Total NADPH concentration was calculated from an internal standard curve and normalised to protein concentration.

ROS detection. MitoSOX Red reagent (ThermoFisher Scientific) was utilised to detect mitochondrialspecific ROS accumulation, using DAPI as a viability marker counter-stain. H1299 cells were seeded in 24-well plates and exposed to siRNA TKT knockdown for $78 \mathrm{~h}$ before treatment with APR-246 $(25 \mu \mathrm{M}) 18 \mathrm{~h}$ prior to ROS detection. Complete media containing $5 \mu \mathrm{M}$ MitoSOX Red reagent was applied to cells and incubated at $37^{\circ} \mathrm{C}$ for $30 \mathrm{~min}$ prior to analysis. Media was removed and replaced with media containing $1 \mu \mathrm{g} / \mathrm{mL}$ DAPI and incubated in the dark for $15 \mathrm{~min}$ at room temperature. Cells were then washed three times, trypsinised and resuspended in PBS supplemented with 1\% v/v FBS and 25 mM EDTA before ROS-induced fluorescence was quantified using the FACSymphony (BD Biosciences).

Cellular proliferation and viability assays. Cellular proliferation was assessed using a microscopybased live cell imaging system (Incucyte FLR, Essen BioScience). Cells were seeded in 96-well plates and treated with indicated doses of APR-246 or vehicle for $72 \mathrm{~h}$. Cells were imaged every $24 \mathrm{~h}$. The confluency of APR246 treated wells was normalised to pre-treatment reading and compared with vehicle treatment. To assess cell viability, AlamarBlue (Life Technologies) fluorometric assays were conducted as per established protocols ${ }^{45}$. Following treatments, $20 \mu \mathrm{L}$ of $2 \% \mathrm{v} / \mathrm{v}$ AlamarBlue was added to each well and incubated for $2 \mathrm{~h}$ at $37^{\circ} \mathrm{C}$. Fluorescence was measured using a FLUOstar OPTIMA microplate reader (BMG Labtech) at an excitation of $540 \mathrm{~nm}$ and an emission of $590 \mathrm{~nm}$. Results were normalised to vehicle-treated wells.

APR-246 dose responses, antioxidant rescue and NAPDH inhibition. Cells were seeded at $2 \times 10^{3}$ cells/well in 96-well plates, exposed to siRNA TKT knockdown or non-targeting control and allowed to adhere overnight. The following day, media was replaced with fresh media supplemented with APR-246 or vehicle control. To determine GI50 values, cells were exposed to a range of concentrations of APR-246 (1 to $100 \mu \mathrm{M})$ for $72 \mathrm{~h}$ and assayed using AlamarBlue. For antioxidant rescue, after $24 \mathrm{~h}$ of siRNA TKT knockdown, cells were treated with $5 \mathrm{mM} \mathrm{N}$-acetylcysteine (NAC) or $15 \mu \mathrm{M}$ APR-246, individually or in combination, for $72 \mathrm{~h}$ before assaying with AlamarBlue. For NADPH inhibition, after $24 \mathrm{~h}$ of siRNA TKT knockdown, cells were treated with $10 \mu \mathrm{M} 6$-aminonicotinamide (6-AN) or vehicle control in combination with a range of APR-246 concentrations for $72 \mathrm{~h}$ before assaying with AlamarBlue. APR-246 dose responses both with and without NADPH inhibition were repeated in cells overexpressing TKT or RFP control, in the aforementioned manner, without exposure to siRNA.

Colony formation assay. Cells in six-well plates were treated with $50 \mu \mathrm{M}$ APR-246 for 5, 10, 15, 20 or $24 \mathrm{~h}$, then typsinised and reseeded in six-well plates, $2 \times 10^{3}$ cells per well and grown for 7 days. Cell colonies were stained with crystal violet $(0.5 \% \mathrm{w} / \mathrm{v})$ containing methanol $(11 \% \mathrm{v} / \mathrm{v})$ for fixing, rinsed in water, air-dried and digitally scanned. Discrete colonies of $>50$ cells were counted using MetaMorph software (Molecular Devices) and expressed as a percentage of vehicle treatment.

Data analysis and statistics. Data were analysed using ANOVA with Dunnet's multiple comparison post-test or Student's $t$-test as indicated. Correlation between two groups was evaluated by the Pearson's test as indicated. GI50 concentration of APR-246 was determined using the Hill equation. Coefficient of interaction (CI) between drugs (APR-246 or erastin) and TKT knockdown (TKT siRNA) or knockout (TKT sgRNA) was 
calculated based on a Bliss independence mode ${ }^{46}$ as described by others ${ }^{47}$. A CI $<0.8$ was defined as synergistic, 0.8 to 1.2 as additive and $>1.2$ as antagonistic. All statistical analyses were performed with Prism 7.02 software (GraphPad) with significance level $\alpha=0.05$.

\section{Data availability}

All primary data can be made available upon reasonable request.

Received: 16 April 2020; Accepted: 10 February 2021

Published online: 24 February 2021

\section{References}

1. Duffy, M. J., Synnott, N. C. \& Crown, J. Mutant p53 as a target for cancer treatment. Eur. J. Cancer 83, 258-265. https://doi. org/10.1016/j.ejca.2017.06.023 (2017).

2. Kastenhuber, E. R. \& Lowe, S. W. Putting p53 in context. Cell 170, 1062-1078. https://doi.org/10.1016/j.cell.2017.08.028 (2017).

3. Lane, D. P. Cancer p53, guardian of the genome. Nature 358, 15-16. https://doi.org/10.1038/358015a0 (1992).

4. Bieging, K. T. \& Attardi, L. D. Deconstructing p53 transcriptional networks in tumor suppression. Trends Cell Biol 22, 97-106. https://doi.org/10.1016/j.tcb.2011.10.006 (2012).

5. Song, H., Hollstein, M. \& Xu, Y. p53 gain-of-function cancer mutants induce genetic instability by inactivating ATM. Nat. Cell Biol. 9, 573. https://doi.org/10.1038/ncb1571 (2007).

6. Hanel, W. et al. Two hot spot mutant p53 mouse models display differential gain of function in tumorigenesis. Cell Death Differ 20, 898-909. https://doi.org/10.1038/cdd.2013.17 (2013).

7. Kollareddy, M. et al. Regulation of nucleotide metabolism by mutant p53 contributes to its gain-of-function activities. Nat. Commun. 6, 7389. https://doi.org/10.1038/ncomms8389 (2015).

8. Sankala, H., Vaughan, C., Wang, J., Deb, S. \& Graves, P. R. Upregulation of the mitochondrial transport protein, Tim50, by mutant p53 contributes to cell growth and chemoresistance. Arch. Biochem. Biophys. 512, 52-60. https://doi.org/10.1016/j.abb.2011.05.005 (2011).

9. Scian, M. J. et al. Tumor-derived p53 mutants induce NF-kappaB2 gene expression. Mol. Cell Biol. 25, 10097-10110. https://doi. org/10.1128/MCB.25.22.10097-10110.2005 (2005).

10. Solomon, H., Madar, S. \& Rotter, V. Mutant p53 gain of function is interwoven into the hallmarks of cancer. J. Pathol. 225, 475-478. https://doi.org/10.1002/path.2988 (2011).

11. Lisek, K., Campaner, E., Ciani, Y., Walerych, D. \& Del Sal, G. Mutant p53 tunes the NRF2-dependent antioxidant response to support survival of cancer cells. Oncotarget 9, 20508-20523. https://doi.org/10.18632/oncotarget.24974 (2018).

12. Bykov, V. J. N. et al. Restoration of the tumor suppressor function to mutant p53 by a low-molecular-weight compound. Nat. Med. 8, 282-288 (2002).

13. Bykov, V. J. N., Eriksson, S. E., Bianchi, J. \& Wiman, K. G. Targeting mutant p53 for efficient cancer therapy. Nat. Rev. Cancer 18, 89. https://doi.org/10.1038/nrc.2017.109 (2017).

14. Lambert, J. M. et al. PRIMA-1 reactivates mutant p53 by covalent binding to the core domain. Cancer Cell 15, 376-388. https:// doi.org/10.1016/j.ccr.2009.03.003 (2009).

15. Mohell, N. et al. APR-246 overcomes resistance to cisplatin and doxorubicin in ovarian cancer cells. Cell Death Dis. 6, e1794. https ://doi.org/10.1038/cddis.2015.143 (2015).

16. Liu, D. S. et al. Inhibiting the system xC(-)/glutathione axis selectively targets cancers with mutant-p53 accumulation. Nat. Commun. 8, 14844. https://doi.org/10.1038/ncomms14844 (2017).

17. Peng, X. et al. APR-246/PRIMA-1MET inhibits thioredoxin reductase 1 and converts the enzyme to a dedicated NADPH oxidase. Cell Death Dis. 4, e881. https://doi.org/10.1038/cddis.2013.417 (2013).

18. Haffo, L. et al. Inhibition of the glutaredoxin and thioredoxin systems and ribonucleotide reductase by mutant p53-targeting compound APR-246. Sci. Rep. 8, 12671-12671. https://doi.org/10.1038/s41598-018-31048-7 (2018).

19. Muller, P. A. \& Vousden, K. H. Mutant p53 in cancer: New functions and therapeutic opportunities. Cancer Cell 25, 304-317. https ://doi.org/10.1016/j.ccr.2014.01.021 (2014).

20. Yue, X. et al. Mutant p53 in cancer: Accumulation, gain-of-function, and therapy. J. Mol. Biol. 429, 1595-1606. https://doi. org/10.1016/j.jmb.2017.03.030 (2017).

21. Kalo, E. et al. Mutant p53R273H attenuates the expression of phase 2 detoxifying enzymes and promotes the survival of cells with high levels of reactive oxygen species. J. Cell Sci. 125, 5578-5586. https://doi.org/10.1242/jcs.106815 (2012).

22. Couto, N., Wood, J. \& Barber, J. The role of glutathione reductase and related enzymes on cellular redox homoeostasis network. Free Radic. Biol. Med. 95, 27-42. https://doi.org/10.1016/j.freeradbiomed.2016.02.028 (2016).

23. Pastore, A., Federici, G., Bertini, E. \& Piemonte, F. Analysis of glutathione: Implication in redox and detoxification. Clin. Chim. Acta 333, 19-39. https://doi.org/10.1016/s0009-8981(03)00200-6 (2003).

24. Dong, Y. \& Wang, M. Knockdown of TKTL1 additively complements cisplatin-induced cytotoxicity in nasopharyngeal carcinoma cells by regulating the levels of NADPH and ribose-5-phosphate. Biomed. Pharmacother. 85, 672-678. https://doi.org/10.1016/j. biopha.2016.11.078 (2017).

25. Xu, I. M. et al. Transketolase counteracts oxidative stress to drive cancer development. Proc. Natl. Acad. Sci. U.S.A. 113, E725-734. https://doi.org/10.1073/pnas.1508779113 (2016).

26. Mitsuishi, Y. et al. Nrf2 redirects glucose and glutamine into anabolic pathways in metabolic reprogramming. Cancer Cell 22, 66-79. https://doi.org/10.1016/j.ccr.2012.05.016 (2012).

27. Frederiks, W. M. et al. Elevated activity of the oxidative and non-oxidative pentose phosphate pathway in (pre)neoplastic lesions in rat liver. Int. J. Exp. Pathol. 89, 232-240. https://doi.org/10.1111/j.1365-2613.2008.00582.x (2008).

28. Phelps, R. M. NCI-navy medical oncology branch cell line data base. J. Cell. Biochem. Suppl. 24, 32 (1996).

29. Hector, A. et al. Establishment and characterization of a bona fide Barrett esophagus-associated adenocarcinoma cell line. Cancer Biol. Ther. 7, 1753-1755. https://doi.org/10.4161/cbt.7.11.6723 (2014).

30. Panieri, E. \& Santoro, M. M. ROS homeostasis and metabolism: A dangerous liason in cancer cells. Cell Death Dis. 7, e2253. https ://doi.org/10.1038/cddis.2016.105 (2016).

31. Reinhold, W. C., Sunshine, M., Varma, S., Doroshow, J. H. \& Pommier, Y. Using cell miner 1.6 for systems pharmacology and genomic analysis of the NCI-60. Clin. Cancer Res. 21, 3841-3852. https://doi.org/10.1158/1078-0432.Ccr-15-0335 (2015).

32. Reinhold William, C. et al. Cell miner: A relational database and query tool for the NCI-60 cancer cell lines. BMC Genom. 10(1), 277. https://doi.org/10.1186/1471-2164-10-277 (2009).

33. Patra, K. C. \& Hay, N. The pentose phosphate pathway and cancer. Trends Biochem. Sci. 39, 347-354. https://doi.org/10.1016/j. tibs.2014.06.005 (2014).

34. Heintze, J., Costa, J. R., Weber, M. \& Ketteler, R. Ribose 5-phosphate isomerase inhibits LC3 processing and basal autophagy. Cell Signal 28, 1380-1388. https://doi.org/10.1016/j.cellsig.2016.06.015 (2016). 
35. Ciou, S. C. et al. Ribose-5-phosphate isomerase A regulates hepatocarcinogenesis via PP2A and ERK signaling. Int. J. Cancer 137, 104-115. https://doi.org/10.1002/ijc.29361 (2015).

36. Weisz, L. et al. Transactivation of the EGR1 gene contributes to mutant p53 gain of function. Can. Res. 64, 8318-8327. https://doi. org/10.1158/0008-5472.Can-04-1145 (2004).

37. Zhou, G. et al. Gain-of-function mutant p53 promotes cell growth and cancer cell metabolism via inhibition of AMPK activation. Mol. Cell. 54, 960-974. https://doi.org/10.1016/j.molcel.2014.04.024 (2014).

38. Zhang, C. et al. Tumour-associated mutant p53 drives the Warburg effect. Nat. Commun. 4, 2935. https://doi.org/10.1038/ncomm s3935 (2013).

39. Freed-Pastor, W. A. et al. Mutant p53 disrupts mammary tissue architecture via the mevalonate pathway. Cell 148, 244-258. https ://doi.org/10.1016/j.cell.2011.12.017 (2012).

40. Diehn, M. et al. Association of reactive oxygen species levels and radioresistance in cancer stem cells. Nature 458, 780-783. https ://doi.org/10.1038/nature07733 (2009).

41. Gorrini, C., Harris, I. S. \& Mak, T. W. Modulation of oxidative stress as an anticancer strategy. Nat. Rev. Drug Discov. 12, 931-947. https://doi.org/10.1038/nrd4002 (2013).

42. Hothersall, J. S., Gordge, M. \& Noronha-Dutra, A. A. 97 (ELSEVIER SCIENCE DIVISION, Netherlands, 1998).

43. Tyson, R. L., Perron, J. \& Sutherland, G. R. 1845 (RAPID COMMUNICATIONS OXFORD LTD, Great Britain, 2000).

44. Jiang, P. et al. p53 regulates biosynthesis through direct inactivation of glucose-6-phosphate dehydrogenase. Nat. Cell Biol. 13, 310-316. https://doi.org/10.1038/ncb2172 (2011).

45. Liu, D. S. et al. APR-246 potently inhibits tumour growth and overcomes chemoresistance in preclinical models of oesophageal adenocarcinoma. Gut 64, 1506-1516. https://doi.org/10.1136/gutjnl-2015-309770 (2015).

46. Bliss, C. I. The toxicity of poisons applied jointly. Ann. Appl. Biol. 26, 585-615 (1939).

47. Yan, S. et al. The RNA polymerase I transcription inhibitor CX-5461 cooperates with topoisomerase 1 inhibition by enhancing the DNA damage response in homologous recombination-proficient high-grade serous ovarian cancer. Br. J. Cancer https://doi. org/10.1038/s41416-020-01158-z (2020).

\section{Acknowledgements}

We thank the following Peter MacCallum Cancer Centre core facilities: Flow Cytometry, Victorian Centre for Functional Genomics. This research was supported by a National Health and Medical Research Council (NHMRC) Project Grant \#APP1120293 (W.A.P. and N.J.C.) and the Australian Cancer Research Foundation (for the Peter MacCallum Cancer Centre Flow Cytometry facility and Victorian Centre for Functional Genomics). J.V.M. and K.M.F. are supported by an Australian Government Research Training Program (RTP) Scholarship. N.J.C. is supported by a Fellowship (MCRF16002) from the Department of Health and Human Services acting through the Victorian Cancer Agency, Victoria, Australia.

\section{Author contributions}

J.V.M., B.Z., K.M.F., S.D. and N.J.C. designed and performed experiments, and analysed data. JVM prepared figures and wrote the main manuscript text. N.J.C. conceived the study. J.V.M., W.A.P. and N.J.C. edited the manuscript. J.V.M., K.M.F., S.D., W.A.P. and N.J.C. reviewed the final manuscript.

\section{Competing interests}

The authors declare no competing interests.

\section{Additional information}

Supplementary Information The online version contains supplementary material available at https://doi. org/10.1038/s41598-021-83979-3.

Correspondence and requests for materials should be addressed to N.J.C.

Reprints and permissions information is available at www.nature.com/reprints.

Publisher's note Springer Nature remains neutral with regard to jurisdictional claims in published maps and institutional affiliations.

(i) Open Access This article is licensed under a Creative Commons Attribution 4.0 International cc) License, which permits use, sharing, adaptation, distribution and reproduction in any medium or format, as long as you give appropriate credit to the original author(s) and the source, provide a link to the Creative Commons licence, and indicate if changes were made. The images or other third party material in this article are included in the article's Creative Commons licence, unless indicated otherwise in a credit line to the material. If material is not included in the article's Creative Commons licence and your intended use is not permitted by statutory regulation or exceeds the permitted use, you will need to obtain permission directly from the copyright holder. To view a copy of this licence, visit http://creativecommons.org/licenses/by/4.0/.

(c) The Author(s) 2021 